ENSAYOS

\title{
Roles y actores del sector Defensa a la luz de la Ley 21.255, Ley Chilena Antártica
}

\author{
Roles and actors of the Defense sector through the 21.255 Law, \\ Chilean Antarctic Law
}

\author{
Pablo Eduardo Urquízar Muñoz y Bárbara Andrea Cortés Cabrera \\ Investigadores independientes, Chile
}

\begin{abstract}
RESUMEN Se analizan los roles y funciones de cada uno de los organismos e instituciones del sector Defensa a la luz de la nueva Ley Chilena Antártica y del Sistema de Tratado Antártico, desde la conducción política, operativa conjunta y operativa institucional de la Defensa en la Antártica, además de otras funciones y sus principales desafíos.
\end{abstract}

PALABRAS CLAVE Defensa, Fuerzas Armadas, roles, Antártica.

ABSTRACT The roles and functions of each of the Defense sector organizations and institutions are analyzed through the new Chilean Antarctic Law and the Antarctic Treaty System, from the political leadership, joint operations and institutional operations of Defense in Antarctica, in addition to other functions and their main challenges.

KEYWORDS Defense, Armed Forces, roles, Antarctica.

\section{Introducción}

Chile tiene una dimensión tricontinental con presencia en Oceanía, en América y en la Antártica. Precisamente sobre este último continente, nuestro país tiene derechos soberanos fundados sobre bases históricas, geográficas y jurídicas, ejerciendo y armonizándolos con su calidad de miembro del Tratado Antártico ${ }^{1}$ de 1959.

1. Decreto 361, promulga el Tratado Antártico. Ministerio de Relaciones Exteriores. Santiago, Chile. Publicado en el Diario Oficial el 14 de julio de 1961. 
La privilegiada posición geográfica que la porción americana de nuestro país mantiene con la Antártica, a tan solo $974 \mathrm{~km}$ de distancia, ha consolidado históricamente a Chile como la puerta de entrada al Continente Blanco. La participación y presencia histórica de la defensa nacional a través de las Fuerzas Armadas en el continente ha contribuido de un modo relevante a nuestra soberanía antártica. Así, en 1916 se efectuó el primer rescate marítimo en ese continente por medio de la escampavía Yelcho, al mando del piloto Luis Pardo, la que rescató a la expedición inglesa de sir Ernest Shackleton. En 1947 se realizó la primera expedición nacional que permitió la fundación de la primera base nacional denominada «Soberanía», ${ }^{2}$ actualmente Base Prat, bajo responsabilidad de la Armada de Chile. Al año siguiente, en 1948, se inauguró la Base O’Higgins, a cargo del Ejército de Chile.

Posteriormente, en 1951 la Fuerza Aérea de Chile inauguró la Base Gabriel González Videla, y en 1955 fundó la Base Pedro Aguirre Cerda, en la isla volcánica Decepción, que durante algunos años estuvo encargada de recopilar información meteorológica. Sin embargo, producto de una erupción volcánica fue destruida.

Tras ello, en 1969 se reactivó el Centro Meteorológico Antártico mediante la inauguración de la Base Frei. En 1980 se construyó y entró en operación el Aeródromo Teniente Marsh que permitió la operación de vuelos en forma permanente, a cargo de la Fuerza Aérea de Chile y de la Dirección General de Aeronáutica Civil (DGAC). Otro hito importante de la presencia de las Fuerzas Armadas en la Antártica es la instalación de Villa Las Estrellas, que contaba con viviendas para funcionarios de la Fuerza Aérea de Chile, de la DGAC, del Instituto Antártico Chileno y profesores civiles.

Adicionalmente, a partir del año 1987, se sumaron a este complejo la Gobernación Marítima y Capitanía de Puerto Bahía Fildes de la Armada de Chile, y posteriormente, en 1995, la Base Profesor Julio Escudero, del Instituto Antártico Chileno (INACH). Sin lugar a dudas, todos estos hechos han convertido a Chile en la puerta de entrada de muchos programas extranjeros que operan en el sector.

De conformidad a la Constitución Política de la República, las Fuerzas Armadas existen "para la defensa de la patria y son esenciales para la seguridad nacional». ${ }^{3}$ Por su parte, el Libro Blanco de la Defensa del 2017 establece las cinco áreas de misión de las FF. AA.: la defensa; cooperación internacional; emergencia nacional y protección civil; contribución al desarrollo del Estado; y seguridad e intereses territoriales. Esta última expresamente incluye la subárea de «mantenimiento y consolidación de la presencia nacional en la Antártica» (Ministerio de Defensa Nacional, 2018: 115), cuestión fundamental para la protección y fortalecimiento de los derechos soberanos antárticos.

2. Desde esa fecha Chile ha tenido una presencia física ininterrumpida.

3. Constitución Política de la República de Chile, artículo 101. 
El pasado 17 de septiembre, se publicó la Ley 21.255, Ley Chilena Antártica, ${ }^{4}$ viniendo a modernizar la normativa antártica. ${ }^{5}$ El objetivo en el marco de su elaboración, como se señaló en la historia fidedigna de la referida ley, fue «por una parte, impulsar la activa participación en la gestación, implementación y desarrollo de un régimen antártico efectivo, a partir del Tratado Antártico de 1959, y por otra, adecuar las normas de derecho interno que rigen, las que requieren actualizarse, según las nuevas exigencias propias del marco internacional que rige en el país, así como para que expresen, de manera clara y eficaz, la forma en que Chile ejerce sus competencias y asume sus obligaciones en el marco del Tratado Antártico» (BCN, 2020: 145). Así, la nueva Ley Chilena Antártica presenta diversos desafíos para el sector Defensa a través de distintos actores, tales como: el Ministerio de Defensa Nacional (MDN), las FF. AA., el Estado Mayor Conjunto (EMCO), la Subsecretaría de Defensa y la Dirección General del Territorio Marítimo y de Marina Mercante (Directemar), entre otros.

En el presente artículo, analizaremos los roles y funciones de cada uno de los organismos e instituciones del sector Defensa a la luz de la nueva Ley Chilena Antártica y del Sistema del Tratado Antártico, desde la conducción política, operativa conjunta y operativa institucional de la defensa en la Antártica, además de otras funciones y sus principales desafíos.

\section{La Defensa y el Sistema del Tratado Antártico}

El Sistema del Tratado Antártico (STA), comprende, el Tratado Antártico (TA) suscrito en Washington el 1 de diciembre de 1959, su Protocolo sobre Protección del Medio Ambiente, ${ }^{6}$ suscrito en Madrid el 4 de octubre de 1991, y las Recomendaciones, Medidas, Decisiones y Resoluciones vigentes aprobadas en las Reuniones Consultivas del Tratado Antártico; además, la Convención para la Conservación de las Focas Antárticas, suscrita en Londres el 28 de diciembre de 1972; y la Convención para la Conservación de los Recursos Vivos Marinos Antárticos, suscrita en Canberra el 11 de septiembre de 1980, y las medidas en vigor acordadas por la comisión de esta última. ${ }^{7}$

La piedra angular del Sistema del Tratado Antártico es el Tratado Antártico, cuya

4. Su nombre oficial es Estatuto Chileno Antártico, pero con el objeto de diferenciarlo del Decreto 298, de 1956, que aprueba el Estatuto del Territorio Antártico Chileno - que sigue vigente-, y para efectos explicativos, se da el nombre de Ley Chilena Antártica.

5. Especialmente la Ley 11.846, de 1955, y el Decreto 298, de 1956, que aprueba el Estatuto del Territorio Antártico Chileno.

6. Decreto 396, promulga el Protocolo al Tratado Antártico sobre Protección del Medio Ambiente. Ministerio de Relaciones Exteriores. Santiago, Chile. Publicado en el Diario Oficial el 18 de febrero de 1998.

7. Numeral 4 del artículo 5 de la Ley 21.255, Estatuto Chileno Antártico. 
regla de consenso sirve de base para la toma de decisiones que rige el Sistema, en que «cada Estado nación protege sus derechos e intereses; y contribuye a proyectar una posición dentro del sistema internacional general» (Rodríguez Márquez y Puig Morales, 2007: 45). Al respecto, cabe recordar que este tratado fue suscrito en 1959 por Argentina, Australia, Bélgica, Chile, Estados Unidos, Francia, Japón, Noruega, Nueva Zelandia, Reino Unido, Sudáfrica, y la Unión Soviética (hoy Rusia). Estos países adquirieron automáticamente la condición de miembros consultivos.

De estos miembros signatarios, los Estados que mantienen reclamaciones territoriales en la Antártica son Argentina, Australia, Chile, Francia, Noruega, Nueva Zelandia y el Reino Unido. También existen otros Estados que, sin reconocer soberanía de otros países, han representado su derecho a formular reclamaciones en la región, como es el caso de Estados Unidos y Rusia. En otra vereda, de los países signatarios, Bélgica, Japón y Sudáfrica no reclaman territorios, pero tampoco reconocen las reivindicaciones de los otros signatarios.

En cuanto a las actividades que puedan realizarse en la Antártica, el artículo I del Tratado Antártico señala en su numeral 1 que «la Antártica se utilizará exclusivamente para fines pacíficos». Por ello «se prohíbe, entre otras, toda medida de carácter militar, tal como el establecimiento de bases y fortificaciones militares, la realización de maniobras militares, así como los ensayos de toda clase de armas.» Por su parte, el numeral 2 del mismo artículo establece que «el presente Tratado no impedirá el empleo de personal o equipo militares para investigaciones científicas o para cualquier otro fin pacífico». Como bien se ha recordado, «al discutirse este artículo hubo detenido intercambio de ideas por parte de diferentes países, con el propósito de aclarar y fortalecer el principio de desmilitarización que en el proyecto original aparecía en forma menos completa» (Mora, 2019: 5).

En relación a las bases y fortificaciones militares, «para que no cupiera dudas sobre [la] posición [de Chile] tanto con respecto al uso pacífico de nuestras Fuerzas Armadas en el Territorio Antártico Chileno, como con respecto a la subsistencia [del] derecho de legítima defensa en dicho territorio, [se incluyó] en el acta de la última sesión del comité plenario celebrada el 30 de noviembre, la siguiente declaración: «La delegación de Chile interpreta el párrafo II del artículo I en el sentido de que Chile podrá mantener $y$ aprovisionar sus bases antárticas actuales y futuras, por intermedio de sus fuerzas armadas regulares y que el conjunto de las disposiciones del referido artículo I en nada menoscaba el derecho de legítima defensa, individual y colectiva, consagrado explícitamente en la Carta de las Naciones Unidas»» (Mora, 2019: 6).

En la actualidad, Chile mantiene una presencia efectiva en la Antártica y en las cercanías del Polo Sur mediante 11 bases antárticas permitiendo, entre otras, la investigación científica, la protección del medioambiente antártico, la colaboración internacional y el reforzamiento de nuestra presencia soberana. La defensa juega un rol fundamental en el vínculo y presencia permanente al mantener 8 bases antárticas; 4 
son bases permanentes (Base Presidente Frei, Base O’Higgins, Base Prat, y Gobernación Marítima Bahía Fildes) y 4 bases estivales (Base González Videla, Base Teniente Carvajal, Base General Boonen y la Estación Polar Conjunta Glaciar Unión, a 1.200 $\mathrm{km}$ del Polo Sur).

\section{La Defensa y los objetivos de la Ley Chilena Antártica}

La nueva Ley Chilena Antártica, establece en su artículo 1, cinco objetivos respecto de los cuales el sector Defensa tiene, en distintos grados, un rol fundamental.

1. «Proteger y fortalecer los derechos soberanos antárticos de Chile, con claros fundamentos geográficos, históricos, diplomáticos y jurídicos».

Este es el primer objetivo de la Ley Chilena Antártica, en línea con la Política Antártica Nacional que establece también como primer objetivo: «proteger los derechos soberanos de Chile sobre el Territorio Chileno Antártico». ${ }^{8}$ Recordemos que forman el Territorio Chileno Antártico, de acuerdo a la nueva Ley 21.255, «todas las tierras, islas, islotes, arrecifes, glaciares (pack-ice) y demás, conocidos y por conocer, existentes dentro de los límites del casquete constituido por los meridianos $53^{\circ}$ longitud Oeste de Greenwich y $90^{\circ}$ longitud Oeste de Greenwich, conforme lo dispuso el Decreto Supremo núm. 1.747, de 1940, del Ministerio de Relaciones Exteriores. Asimismo, forman la Antártica Chilena o Territorio Chileno Antártico las barreras de hielo, el mar territorial, la zona contigua, la zona económica exclusiva, la plataforma continental, la plataforma continental extendida y todos los espacios marítimos que le correspondan de conformidad con el Derecho Internacional». ${ }^{9}$ En este sentido, se reconoce que «el resguardo y la promoción de los derechos soberanos de Chile en la Antártica son, y continuarán siendo, un objetivo prioritario y permanente de la política exterior de nuestro país». ${ }^{10}$

Es importante recordar, como ya se señaló, que antes de la entrada en vigencia del TA, siete países habían reclamado derechos de soberanía en la Antártica dentro de los cuáles se encuentra nuestro país. Al respecto, dicho tratado establece que «ninguna disposición del presente Tratado se interpretará: a) como una renuncia, por cualquiera de las Partes Contratantes, a sus derechos de soberanía territorial o a las reclamaciones territoriales en la Antártica, que hubiere hecho valer precedentemente; b) como una renuncia o menoscabo, por cualquiera de las Partes Contratantes». ${ }^{11}$ Asimismo, el numeral 2 del mismo artículo consagra que «no se harán nuevas recla-

8. Decreto 56, aprueba Política Antártica Nacional. Ministerio de Relaciones Exteriores. Santiago, Chile. Publicado en el Diario Oficial el 12 de diciembre de 2017. Capítulo II.

9. Artículo II del Tratado Antártico.

10. Véase nota 8.

11. Artículo IV del Tratado Antártico. 
maciones de soberanía territorial en la Antártica, ni se ampliarán las reclamaciones anteriores hechas vales, mientras el presente Tratado se halle en vigencia».

En armonía con lo anterior, el artículo 4 de la Ley Chilena Antártica consagra que «la soberanía chilena se ejercerá con pleno respeto a las normas del Derecho Internacional y a los compromisos internacionales del Estado de Chile que se encuentren vigentes, en el marco del Sistema del Tratado Antártico, y en conformidad con lo dispuesto en el ordenamiento jurídico chileno.»

De acuerdo al Libro de la Defensa Nacional, la soberanía territorial implica la capacidad de la autoridad política de hacer prevalecer la jurisdicción exclusiva del Estado sobre un territorio determinado. En la actualidad, esta perspectiva incluye una mirada del territorio de carácter multidimensional, y en el que las FF. AA. contribuyen a mantener la integridad e integración territorial del país y a participar en la cooperación transfronteriza conforme a los compromisos de la comunidad internacional (Ministerio de Defensa Nacional, 2018: 34). Este enfoque multidimensional ha sido producto del tránsito de una mirada disgregada del territorio hacia una visión integrada del territorio procurando equilibrar las políticas e instituciones en la planificación de la Defensa Nacional. La Política de Defensa responde a los desafíos impuestos por el enfoque multidimensional del territorio nacional y del derecho internacional (Ministerio de Defensa Nacional, 2018: 34). De esta manera, según expresa, «Chile mantiene en forma irrenunciable sus derechos de soberanía territorial en la Antártica y, al amparo del reconocimiento consignado en su artículo IV, el Tratado Antártico protege la situación jurídica del Territorio Chileno Antártico» (Ministerio de Defensa Nacional, 2018: 35).

Asimismo, el Plan Estratégico Antártico 2020-2024, establece como objetivo general «consolidar y fortalecer los derechos soberanos que Chile mantiene y perfecciona a través de la presencia efectiva, protección del medio ambiente, desarrollo de una ciencia beneficiosa para el país, implementación de una logística sustentable y operaciones antárticas seguras con la ejecución de actividades económicas permitidas por el Tratado Antártico» (Ministerio de Relaciones Exteriores, 2020: 5). Para ello, sin lugar a dudas el sector Defensa cumple un rol esencial con su presencia efectiva e ininterrumpida.

2. «Establecer los principios conforme a los cuales el Estado de Chile, a través de los órganos competentes, conduce la política antártica y ejerce sus competencias en materia antártica».

El segundo objetivo de la Ley Chilena Antártica se refiere a los lineamientos fundamentales y la institucionalidad antártica chilena para ello. Sin perjuicio de la conducción del Ministerio de Relaciones Exteriores, las diversas funciones de las instituciones y organismos del sector Defensa, como se verá más adelante, cumplen un rol crucial en la Política Antártica Nacional, en el Consejo de Política Antártica, como 
operadores antárticos estatales, en la protección del medioambiente antártico y en el apoyo a la ciencia e investigación científica, entre otras.

3. «Promover la protección y el cuidado del medioambiente antártico y sus ecosistemas dependientes y asociados, así como su condición de reserva natural, dedicada a la paz y a la investigación científica, a través del reforzamiento y profundización del Sistema del Tratado Antártico».

Recordemos que dentro del STA, se encuentra el Protocolo sobre Protección del Medio Ambiente, suscrito en Madrid el 4 de octubre de 1991, el cual establece en el numeral 1 de su artículo 3 que «la protección del medio ambiente antártico y los ecosistemas dependientes y asociados, así como del valor intrínseco de la Antártica, incluyendo sus valores de vida silvestre y estéticos y su valor como área para la realización de investigaciones científicas, en especial las esenciales para la comprensión del medio ambiente global, deberán ser consideraciones fundamentales para la planificación y realización de todas las actividades que se desarrollen en el área del Tratado Antártico». Este objetivo es plenamente coincidente con el tercer objetivo de la Política Antártica Nacional que establece «proteger y promover el cuidado del medio ambiente antártico, incluyendo el medio ambiente marino y sus ecosistemas dependientes y asociados». A la defensa le corresponde un rol primordial en la fiscalización de ello en la Antártica.

4. «Potenciar y regular las actividades antárticas de Chile, incrementando su calidad de prestador de servicios operativos, logísticos, tecnológicos y científicos antárticos, e incentivando el desarrollo del país ligado a las actividades antárticas, estatales y no estatales».

Esto se encuentra ligado indiscutiblemente al rol de Operador Antártico Estatal que tienen las FF. AA., cuyas funciones específicas en este ámbito se encuentran en el artículo 16 de la Ley Chilena Antártica, sobre el cual se profundiza más adelante.

5. «Fomentar la actividad antártica de Chile, promoviendo el desarrollo social y económico de la Región de Magallanes y de la Antártica Chilena».

Sin lugar a dudas, este objetivo se encuadra en el ámbito del sector Defensa de un modo indirecto vinculado al cumplimiento de los objetivos anteriores que pueden tener como efecto, este último objetivo.

\section{Actores del sector Defensa y roles en la Ley Chilena Antártica}

En cumplimiento de los objetivos establecidos por la Ley 21.255, en línea con los instrumentos ejecutivos en materia antártica para el quehacer en el continente, es menester explicar los roles y funciones que tendrá el sector Defensa y que se enmarcan dentro de los compromisos internacionales. 


\section{Ministerio de Defensa Nacional}

Es el órgano superior de colaboración del Presidente de la República en las funciones de gobierno y administración de la defensa nacional. ${ }^{12}$. La Ley 21.255, consagra al MDN dentro del Título II referido a la «Institucionalidad antártica chilena», específicamente en el artículo 12. El referido artículo viene a precisar por primera vez la función de dicha cartera en relación a la Antártica. Así, establece en su inciso primero que «será competencia específica del Ministerio de Defensa Nacional planificar, coordinar y ejercer la dirección de las actividades antárticas que efectúen las Fuerzas Armadas y entidades dependientes de la defensa nacional». El objetivo primordial de esta regulación, según la historia fidedigna de su establecimiento es «definir expresamente que la actuación de las Fuerzas Armadas en la Antártica debe obedecer las directrices del Ministerio de Defensa Nacional» (BCN, 2020: 69). En concordancia con lo anterior, el inciso final del artículo 10 de la Ley Chilena Antártica, señala que los diversos ministerios y entidades estatales con competencia sectorial en las actividades antárticas coordinarán su labor por medio del Ministerio de Relaciones Exteriores, con excepción de los operadores antárticos dependientes del Ministerio de Defensa Nacional, los cuales se coordinarán a través de dicho Ministerio.

Es el nivel político de la defensa el responsable de "planificar»; «coordinar», y «ejercer la dirección» de las actividades antárticas. La planificación consiste en el plan general, metódicamente organizado para obtener un objetivo determinado en la Antártica. Esto debe vincularse con los Planes Estratégicos Antárticos cuya duración es de 5 años y que conforme al artículo 8 de la Ley 21.255, comprenden «las tareas y acciones concretas que se materializarán mediante el Programa Antártico Nacional». La coordinación consiste en la disposición ordenada de los medios técnicos y humanos del sector Defensa para su actuar en la Antártica. Por su parte, la dirección consiste en la conducción de las instituciones de la defensa hacia los objetivos antárticos fijados. ${ }^{13}$

El inciso segundo del artículo 12 señalado, establece que «el ejercicio de las competencias del Ministerio de Defensa Nacional señaladas [...] y el empleo de su personal o equipo militar, deberán tomar en consideración que la Antártica se utilizará exclusivamente para fines pacíficos y científicos». Esto último en armonía con el artículo I del TA, que consagra la utilización de la Antártica exclusivamente para fines pacíficos sin perjuicio que el Tratado no impedirá el empleo de personal o equipo militar, para investigaciones científicas o para cualquier otro fin pacífico.

12. Artículo 3 de la Ley 20.424, Estatuto Orgánico del Ministerio de Defensa Nacional.

13. Un ejemplo concreto es la Directiva Ministerial para la ejecución de las tareas antárticas de los órganos del sector Defensa en el período 2019-2020. MDN SSD DIV.RR. II/DCTAI (R) Nºo6, de 6 de mayo de 2019 . 
Asimismo, al MDN le corresponde integrar el Consejo de Política Antártica (en adelante CPA), conforme al artículo 52 de la Ley 21.080 que modifica diversos cuerpos legales con el objeto de modernizar el Ministerio de Relaciones Exteriores. El CPA, de acuerdo al artículo 7 de la Ley Chilena Antártica, «es el órgano colegiado de naturaleza interministerial [...], que tiene por función proponer al Presidente de la República las bases políticas, jurídicas, científicas, económicas, medioambientales, logísticas, deportivas, culturales y de difusión de la acción nacional en la Antártica, y proponer los grandes lineamientos de la Política Antártica Nacional».

\section{Subsecretaría de Defensa}

Es el órgano de colaboración del MDN en asuntos de Política de Defensa, debiendo, entre otras materias, coordinar la ejecución de aquella con la Política Antártica Nacional (Sanhueza, 2019: 7). El artículo 16 de la Ley 21.255 incorpora a la Subsecretaría de Defensa, asignándole la función de coordinación entre las FF. AA. «y los demás ministerios con competencias en la materia». De conformidad a la Directiva Ministerial para la Ejecución de las Tareas Antárticas de los Organismos del Sector Defensa, ${ }^{14}$ la Subsecretaría de Defensa desarrolla un conjunto de tareas, tales como: brindar asesoría al MDN en aquellas materias antárticas, particularmente en calidad de miembro del CPA; realizar coordinaciones con operadores antárticos del sector Defensa; coordinar la gestión política externa del sector Defensa en materia Antártica; participar en reuniones internas con la Dirección de Presupuesto, junto a los operadores antárticos, Estado Mayor Conjunto y Subsecretaría para las Fuerzas Armadas, a fin de gestionar el financiamiento de las actividades que realicen dichos operadores en la Antártica; evaluar las políticas y proyectos que pretendan realizarse en la Antártica, priorizando su ejecución de conformidad a la disponibilidad de recursos económicos; realizar seguimiento al desarrollo de los proyectos antárticos financiados por Defensa conforme a los roles que sus operadores efectúan en la Antártica; instruir a los operadores antárticos la implementación de las decisiones, resoluciones y medidas adoptadas en el marco del Sistema del Tratado Antártico; participar en el Programa Nacional de Inspecciones Antárticas que dirige el Ministerio de Relaciones Exteriores con el apoyo de los operadores antárticos; entre otras.

\section{Subsecretaría para las Fuerzas Armadas}

Si bien no está explicitada en la Ley 21.255, cumple un importante rol al ser ésta, de acuerdo al artículo 20 del Estatuto Orgánico del Ministerio de Defensa Nacional, «el

14. Directiva Ministerial para la ejecución de las tareas antárticas de los órganos del sector Defensa en el período 2019-2020. MDN SSD DIV.RR. II/DCTAI (R) núm. oo6, de 6 de mayo de 2019: 3. 
órgano de colaboración del Ministro en aquellas materias que dicen relación con la formulación de políticas y con la gestión de los asuntos y procesos administrativos que el Ministerio de Defensa Nacional y las Fuerzas Armadas requieran para el desarrollo de la fuerza y el cumplimiento de sus funciones».

Así, la Subsecretaría para las Fuerzas Armadas debe asesorar al MDN en materias de seguimiento del gasto en el programa antártico de las instituciones, de conformidad a la Ley de Presupuestos del Sector Público, según corresponda. Asimismo, esta Subsecretaria participa en reuniones de proyección financiera, dirigidas por el EMCO, en el marco del cumplimiento de tareas que aborda el sector Defensa. Recordemos, además, que el artículo 21 de la Ley Chilena Antártica consagra que la Ley de Presupuestos del Sector Público deberá consultar anualmente recursos para financiar la actividad antártica nacional, de acuerdo a lo señalado en el Programa Antártico Nacional a los operadores antárticos estatales.

\section{Estado Mayor Conjunto}

El artículo 25 de la Ley 20.424, Estatuto Orgánico del Ministerio de Defensa Nacional, señala que es «el organismo de trabajo y asesoría permanente del Ministro de Defensa Nacional en materias que tengan relación con la preparación y empleo conjunto de las Fuerzas Armadas». Se introduce en materia Antártica al EMCO con el desafío de realizar la «coordinación operativa y logística entre las Fuerzas Armadas», conforme al artículo 16 de la Ley 21.255. Lo anterior tiene -como se expresó en la historia fidedigna del establecimiento de la Ley 21.255- «dos potencialidades; una de ellas es la coordinación y aunar esfuerzos de las tres instituciones y el Estado Mayor Conjunto; y la otra, llevar este tema a una concepción netamente conjunta que hasta hoy ha funcionado por la sinergia del trabajo de cada una de las instituciones» (BCN, 2020: 48). Se diferencia su rol con la Subsecretaría de Defensa en que la coordinación de esta última dice relación con el nivel político y no al interior de las FF. AA. En la práctica, el EMCO desarrolla la importante misión de asesorar al ministro de Defensa en la materia y coordinar las actividades conjuntas en la Antártica, como también aquellas que sean necesarias en los campos operativos y de capacitación; así, también, en conjunto con la Subsecretaría de Defensa, Subsecretaría para las Fuerzas Armadas, y operadores antárticos del sector Defensa, deben proyectar el presupuesto anual para financiar la realización de actividades antárticas, para ser luego presentado al Ministerio de Hacienda, en las partidas del EMCO; además, participa como miembro del CPA y en los demás Comités en el marco del Sistema del Tratado Antártico; entre otras. $^{15}$

15. Directiva Ministerial para la ejecución de las tareas antárticas de los órganos del sector Defensa en el período 2019-2020. MDN SSD DIV.RR. II/DCTAI (R) núm. oo6, de 6 de mayo de 2019: 4. 


\section{Fuerzas Armadas}

El artículo 1 de la Ley 18.948, Orgánica Constitucional de las Fuerzas Armadas, señala que «las Fuerzas Armadas, dependientes del Ministerio encargado de la Defensa Nacional, están integradas sólo por el Ejército, la Armada y la Fuerza Aérea, constituyen los cuerpos armados que existen para la defensa de la patria, son esenciales para la seguridad nacional y garantizan el orden institucional de la República». Conforme al numeral 11 del artículo 5 de la Ley 21.255, las FF. AA. forman parte de los operadores antárticos estatales junto al INACH. Estos corresponden a «toda institución u organismo público, que organiza y desarrolla actividades operativas, logísticas, científicas o tecnológicas, a ser ejecutadas en la Antártica, contando con las autorizaciones prescritas en esta ley». Es importante mencionar que el artículo 16 de la Ley Chilena Antártica se refiere a estos, señalando que «los operadores antárticos dependientes del Ministerio de Defensa Nacional coordinarán sus actividades a través de ese ministerio, las que se regirán por los objetivos de la Política Antártica Nacional y los planes estratégicos que se elaboren para su cumplimiento». Los ejes rectores de sus roles están en la Política Antártica Nacional y los planes estratégicos respectivos que se elaboren para el cumplimiento de la misma.

Sus principales roles son:

Encargados de las actividades operativas y logísticas del país en la Antártica y de la mantención de las bases. Esto apunta al transporte estratégico desde Chile americano al Territorio Chileno Antártico, así como el sostenimiento de las bases ${ }^{16}$, estaciones ${ }^{17}$ y refugios $^{18}$ (Subsecretaría de Defensa, 2020: 13). Como bien señala el Libro de la Defensa Nacional, nuestro país «cuenta con una importante cantidad de instalaciones antárticas que permiten mantener presencia efectiva, dar apoyo a la actividad científica desarrollada por el INACH, salvaguardar la vida humana en la Antártica y monitorear las medidas medioambientales que protegen a dicho continente» (Ministerio de Defensa Nacional, 2018: 51). En línea con lo anterior, especial atención además puso la Ley 21.255 con la actividad científica, disponiendo que las FF. AA. «prestarán servicios operacionales y apoyo logístico para el Programa Científico Nacional, en la medida de sus capacidades, roles y actividades propias», de acuerdo al inciso tercero de su artículo 16. En la práctica, esto se traduce en «apoyo a la expedición científica antártica del INACH, el uso de laboratorios en instalaciones antárticas de Defensa y el apoyo al traslado terrestre, marítimo y aéreo en la zona donde se requiere la reali-

16. Base Arturo Prat; Base Bernardo O’Higgins; Base Presidente Gabriel González Videla; Base Presidente Eduardo Frei Montalva; Base Científica Profesor Julio Escudero;

17. Estación Marítima Bahía Fildes y Estación Polar Científica Conjunta Glaciar Unión.

18. Refugio Yelcho; Refugio Guillermo Mann; Refugio Luis Risopatrón; Refugio Julio Ripamonti; Refugio Collins y Refugio General Jorge Boonen Rivera. 
zación de la Ciencia» (Subsecretaría de Defensa, 2020: 14). Como bien se ha señalado «sin logística (y en general sin los medios humanos y materiales que despliega la defensa) no hay presencia soberana, ni campaña antártica, ni es posible realizar ciencia en terreno, ni se cumplen los objetivos nacionales al respecto» (Ferrada, 2012: 37).

Encargados de prestar servicios de búsqueda y salvamento en las áreas de responsabilidad en conformidad con los tratados internacionales vigentes. La búsqueda y salvamento se ha definido como «aquellas funciones de supervisión, comunicación, coordinación, búsqueda y salvamento, asistencia médica inicial o evacuación médica en una situación de peligro, mediante la utilización de todo recurso disponible, incluyendo aeronaves, buques y otras embarcaciones e instalaciones que colaboren en las operaciones». ${ }^{19}$ La Fuerza Aérea tiene la responsabilidad en el ámbito aéreo. ${ }^{20}$ El Reglamento de Búsqueda y Salvamento Aéreo establece en su artículo 2.4. que «el Estado de Chile proporciona el servicio de búsqueda y salvamento aéreo durante las veinticuatro horas del día, y esta ayuda se presta a las aeronaves en peligro y a los supervivientes de accidentes de aviación cualquiera sea la nacionalidad de éstos». Por su parte, la Armada tiene una triple misión, a saber:

1.- Organizar y efectuar la búsqueda de las naves, artefactos navales u otros vehículos de transporte en el área marítima de responsabilidad nacional, con el propósito de rescatar a su dotación y pasajeros que se hallen en peligro, a consecuencia de un accidente en el mar. 2.- Cooperar de propia iniciativa o a requerimiento de autoridades o particulares [...] a la búsqueda y el rescate de vidas humanas y materiales no relacionados con las actividades marítimas de orden general, que se encuentren en peligro o perdidos en el mar y zonas lacustres o fluviales, donde existan Autoridades Marítimas y 3.- Efectuar el Control del Tráfico Marítimo y actividades destinadas a combatir la contaminación de las aguas del mar por hidrocarburos u otros elementos nocivos. ${ }^{21}$

Asimismo, es explícita su responsabilidad en la Antártica. ${ }^{22}$

La Ley Chilena Antártica en el artículo 33 se refiere a las acciones de búsqueda y rescate, estableciendo expresamente que «los centros de coordinación de búsqueda y rescate marítimo y aéreo de Punta Arenas y los sub-centros que se establezcan en el Territorio Chileno Antártico serán los encargados de coordinar las acciones respectivas». De igual modo, continúa dicho artículo, «para efectos de facilitar el cumplimiento de su función, todas las naves o aeronaves que zarpen o despeguen desde

19. Decreto Supremo 531, que aprueba «Reglamento de Búsqueda y Salvamento aéreo» DAR-12, Ministerio de Defensa Nacional (1993), capítulo 1, artículo 1.1.

20. Decreto Supremo 531, capítulo 2, artículo 2.3.

21. Decreto Supremo 1.19o, que organiza el Servicio de Búsqueda y Rescate Marítimo, dependiente de la Armada de Chile. Ministerio de Defensa Nacional (1976). Título I.

22. Decreto Supremo 1.190. Título II. 
puertos o aeropuertos chilenos con destino a la Antártica, cualquiera sea su nacionalidad, naturaleza o dependencia, estatales o privadas, científicas, turísticas, pesqueras u otras, deberán informar a las autoridades pertinentes su plan de navegación o vuelo y comunicar su posición durante la travesía, a intervalos de tiempo regular y al menos diariamente, a los centros o subcentros de búsqueda y rescate chileno».

Encargados de la fiscalización del cumplimiento de la disposición relativa a la regulación de las actividades antárticas, de la protección del medioambiente antártico y comisión de infracciones administrativas y delitos medioambientales en la Antártica. El artículo 47 de la Ley 21.255 dispone que «la fiscalización del cumplimiento de las disposiciones de los títulos V, VI y VII de la presente ley y sus reglamentos será ejercida: a) En el continente Antártico por los funcionarios del Ejército, de la Armada, de la Fuerza Aérea y del Instituto Antártico Chileno que se encuentren en dicho territorio». Los títulos V, VI y VII de la Ley Chilena Antártica se refieren, respectivamente, a la regulación de las actividades antárticas, protección y conservación del medioambiente antártico, y a la fiscalización y sanciones. Asimismo, en dicho artículo se consagra que «en el ejercicio de esta función fiscalizadora, los jefes de bases antárticas de cualquiera de estas instituciones tendrán la calidad de ministros de fe». A ésta fiscalización se debe sumar aquella referida a la normativa internacional del Sistema del Tratado Antártico.

Otras funciones no explicitadas. Complementando las funciones expresamente consagradas en la nueva Ley Chilena Antártica, existen otras igual de relevantes no explicitadas (Ministerio de Defensa Nacional, 2018: 50) como mantener la presencia efectiva en territorio antártico, contribuir a la cooperación internacional apoyando programas antárticos de otros países, integrar el CPA a través de los Comandantes en Jefe de cada institución, la mantención de la señalización marítima en Territorio Chileno Antártico, entre otras.

\section{Dirección General del Territorio Marítimo y Marina Mercante}

Es el organismo de alto nivel de la Armada de Chile que tiene por misión cautelar el cumplimiento de las leyes y acuerdos internacionales vigentes, para dar seguridad a la navegación, proteger la vida humana en el mar, preservar el medio ambiente acuático, los recursos naturales marinos y fiscalizar las actividades que se desarrollan en el ámbito marítimo de su jurisdicción, con el propósito de contribuir al desarrollo marítimo de la nación. ${ }^{23}$ Además de su rol general conforme al Decreto Ley 2.222, tiene la función de conocer y sancionar las infracciones específicas en la Ley Chilena Antártica, consagradas en el artículo 48. Esto es, la eliminación de cualquier tipo de

23. Dirección General del Territorio Marítimo y de Marina Mercante. Misión y Visión, disponible en http://bit.ly/34M87IT. 
basura o residuo en la Antártica, sea en el mar, hielo o en tierra y, la descarga en el mar de la Antártica de aguas residuales, en los términos previstos en el Anexo IV del Protocolo al Tratado Antártico sobre Protección del Medio Ambiente. En este sentido, como señaló en la historia fidedigna de su establecimiento, «el territorio chileno antártico es territorio nacional, por tanto, deben regir las normas sobre control de contaminantes en las aguas, cuyo órgano fiscalizador es Directemar, siendo sus decisiones impugnables, como toda decisión administrativa» (BCN, 2020: 365). La novedad en este ámbito radica en que se ha ampliado la competencia de Directemar a conocer de infracciones relativas a eliminación de basura o residuos en el hielo o tierra antártica, principalmente porque a partir de éstas podría devenir la contaminación del mar antártico.

\section{Dirección General de Aeronáutica Civil}

Es un organismo dependiente de la Comandancia en Jefe de la Fuerza Aérea de Chile, mediante el cual el Estado norma y fiscaliza la actividad aérea, tanto nacional como internacional, que se desarrolla dentro del espacio aéreo controlado por nuestro país (Ministerio de Defensa Nacional, 2018: 294). De acuerdo al artículo 1 de la Ley 16.752, que fija organización y funciones y establece disposiciones generales a la Dirección General de Aeronáutica Civil, a ésta, «le corresponderá fundamentalmente la dirección y administración de los aeródromos públicos y de los servicios destinados a la ayuda y protección de la navegación aérea». Si bien no está expresamente mencionado en la nueva Ley Chilena Antártica, su rol es fundamental en el Continente Blanco. De hecho, la DGAC, tiene una regulación especial que tiene por propósito «establecer las reglas de operación que permitan a las aeronaves de Estado y civiles, nacionales y extranjeras operar hacia y desde el Territorio Antártico, especialmente en los Aeródromos Teniente Marsh y Glaciar Unión y/o utilizarlos como alternativa considerando su infraestructura y servicios disponibles». ${ }^{24}$

\section{Desafíos para el sector Defensa que impone la Ley Chilena Antártica}

La entrada en vigencia de la nueva normativa antártica impone a los actores del sector Defensa una serie de desafíos para poder cumplir a cabalidad con los objetivos establecidos por ésta, la Política Antártica Nacional y los Programas Estratégicos Antárticos.

En primer lugar, la conducción política de la defensa representada principalmente por el MDN, debe continuar perfeccionándose e interiorizándose en los temas antár-

24. DAN Antártico 01 «Operaciones aéreas desde y hacia el Territorio Antártico». Dirección General de Aeronáutica Civil, 1 de agosto de 2018, p. 5. 
ticos de manera de poder realmente planificar, coordinar y dirigir en el ámbito de sus competencias antárticas en la forma que prescribe la nueva Ley Chilena Antártica. Para ello la Academia Nacional de Estudios Políticos y Estratégicos cumple un rol fundamental al incorporar a la Antártica dentro de sus programas de formación.

En segundo lugar, el MDN debe armonizar las Directivas Ministeriales y demás instrumentos ejecutivos antárticos a la nueva Ley Chilena Antártica, de acuerdo a la relevancia y prioridades de sus objetivos en consonancia con la Política de Defensa Nacional y las respectivas áreas de misión.

En tercer lugar, el rol de la Subsecretaría de Defensa y del EMCO debe entenderse adecuadamente en el sentido de que, si bien ambos tienen funciones de coordinación, las naturalezas de esas funciones son distintas. La Subsecretaría de Defensa coordina en el orden civil y político. El Estado Mayor Conjunto, por su parte, coordina en el nivel militar y de las FF. AA. Es un desafío que ambos organismos ejerzan sus competencias dentro del ámbito de sus atribuciones para no enredar lo que precisamente se quiere hacer, que es mejorar la coordinación.

De igual modo, en cuarto lugar, constituye un desafío el que las instituciones de las FF. AA. respondan cada vez más a una visión conjunta en la Antártica con el fortalecimiento del EMCO en su rol de coordinación.

En quinto lugar, resulta de vital importancia que, fijándose los objetivos antárticos, los operadores antárticos estatales del MDN, es decir las FF. AA., reciban los recursos adecuados para cumplir sus funciones operativas y logísticas, así como la infraestructura necesaria, a través de las dos principales fuentes de financiamiento que las considera: la Ley de Presupuestos del Sector Público y la Ley 21.174 que establece un nuevo mecanismo de financiamientos de las capacidades estratégicas de la Defensa Nacional, sin descuidar o ver menoscabadas sus otras áreas de misión.

Es importante también, en sexto lugar, que en cuanto al rol fiscalizador de las FF. AA. exista un protocolo conjunto de sus competencias, con la claridad necesaria de manera que la ejecución de sus funciones sea efectiva.

Por último, y no por ello menos importante, se requiere continuar profundizando el respeto, consideración y aplicación del Sistema del Tratado Antártico en el Continente Blanco, entendiendo que las FF. AA. mantienen presencia ininterrumpida en dicho territorio conjugándola con la protección de nuestros derechos soberanos en el Territorio Chileno Antártico.

\section{Referencias}

BCN, Biblioteca del Congreso Nacional (2020). Historia fidedigna de la Ley 21.255. Estatuto Chileno Antártico. 
Ferrada Walker, Luis Valentín (2012). «La defensa nacional y su aporte a la política antártica de Chile». Revista Escenarios Actuales, 17 (3): 29-40. Disponible en http://bit.ly/387Yo31.

Ministerio de Defensa Nacional (2018). Libro de la Defensa Nacional de Chile. Santiago: Ministerio de Defensa Nacional.

Ministerio De Relaciones Exteriores (2020). «Plan Estratégico Antártico 2020-24».

MorA, Marcial (2019). «El Tratado Antártico», Revista Tribuna Internacional, 8 (16). Disponible en http://bit.ly/309MjOZ.

Rodríguez Márquez, Paul y Mario Puig Morales (2007). Chile y sus Intereses en la Antártica. Opciones Políticas y de Seguridad frente a la escasez de recursos hídricos. Santiago: Academia Nacional de Estudios Políticos y Estratégicos.

Sanhueza, Camilo (2019). «Institucionalidad Antártica Chilena». Revista Tribuna Internacional, 8 (15). Disponible en http://bit.ly/2Lc1Mjo.

Subsecretaría de Defensa (2020). «Gestión Antártica de Defensa, roles, actividades y presupuestos, año 2014-2020».

\section{Sobre los autores}

Pablo Eduardo Urquízar Muñoz es abogado. Licenciado en Derecho y Máster en Derecho (LLM), Pontificia Universidad Católica de Chile. Su correo electrónico es pablo.urquizar@gmail.com.

Bárbara Andrea Cortés Cabrera es abogada. Licenciada en Derecho por la Universidad Alberto Hurtado y Máster en Acción Política, Fortalecimiento Institucional y Participación Ciudadana en el Estado de Derecho por la Universidad Francisco de Vitoria. Su correo electrónico es barbara.cortes.cabrera@gmail.com. 
La Revista Tribuna Internacional busca fomentar la reflexión, el debate, el análisis y la comunicación pluralista y con rigor científico en las áreas del derecho internacional público, derecho internacional privado, relaciones internacionales y derecho internacional de los derechos humanos. Los artículos y ensayos son seleccionados mediante revisión de pares externos a la Facultad de Derecho de la Universidad de Chile. Se reciben trabajos en castellano y en inglés.

EDITOR GENERAL

Luis Valentín Ferrada Walker

SITIO WEB

tribunainternacional.uchile.cl

CORREO ELECTRÓNICO

revistatribuna@derecho.uchile.cl

LICENCIA DE ESTE ARTÍ́CULO

Creative Commons Atribución Compartir Igual 4.o Internacional

La edición de textos, el diseño editorial

y la conversión a formatos electrónicos de este artículo

estuvieron a cargo de Tipográfica

(www.tipografica.io) 